\title{
A low-resource reliable pipeline to democratize multi-modal connectome estimation and analysis
}

\author{
Ross Lawrence*, Alex Loftus, Gregory Kiar, Eric W. Bridgeford, William Gray Roncal, Consortium \\ for Reliability and Reproducibility (CoRR), Vikram Chandrashekhar, Disa Mhembere, \\ Sephira Ryman, Xi-Nian Zuo, Daniel S. Margulies, R. Cameron Craddock, Carey E. Priebe, \\ Rex Jung, Vince D. Calhoun, Brian Caffo, Randal Burns, Michael P. Milham, \\ Joshua T. Vogelstein ${ }^{\dagger}$
}

\begin{abstract}
Connectomics - the study of brain networks - provides a unique and valuable opportunity to study the brain. However, research in human connectomics, accomplished via Magnetic Resonance Imaging (MRI), is a resourceintensive practice: typical analysis routines require impactful decision making and significant computational capabilities. Mitigating these issues requires the development of low-resource, easy to use, and flexible pipelines which can be applied across data with variable collection parameters. In response to these challenges, we have developed the MRI to Graphs (m2g) pipeline. $\mathrm{m} 2 \mathrm{~g}$ leverages functional and diffusion datasets to estimate connectomes reliably. To illustrate, $\mathrm{m} 2 \mathrm{~g}$ was used to process $\mathrm{MRI}$ data from 35 different studies $(\approx 6,000$ scans) from 15 sites without any manual intervention or parameter tuning. Every single scan yielded an estimated connectome that followed established properties, such as stronger ipsilateral than contralateral connections in structural connectomes, and stronger homotopic than heterotopic correlations in functional connectomes. Moreover, the connectomes generated by $\mathrm{m} 2 \mathrm{~g}$ are more similar within individuals than between them, suggesting that $\mathrm{m} 2 \mathrm{~g}$ preserves biological variability. $\mathrm{m} 2 \mathrm{~g}$ is portable, and can run on a single CPU with $16 \mathrm{~GB}$ of RAM in less than a couple hours, or be deployed on the cloud using its docker container. All code is available on https://neurodata.io/mri/.
\end{abstract}

1 Introduction Human brain imaging, especially Magnetic Resonance Imaging (MRI), has become a vital tool in both basic and clinical brain science [1], with new analysis techniques being developed frequently. One such area concerns itself with estimation and analysis of connectomes from MRI data, allowing for the use of graph theoretic mathematics and statistics to discern both physiological and functional relationships. A connectome is a comprehensive map of relationships present in the brain. The creation of a connectome revolves around the number of connections, or edges, between different areas of the brain called regions of interest (ROIs). Where the boundaries lie for each of these regions of interest depends on the parcellation method used, as there a many different ways to group regions of the brain. Edges can represent any type of relationships between ROls. For structural connectomes, which are generated using diffusion-weighted MRI scans, edges represent the quantity of white-matter tracks that connect ROls. For functional connectomes, which are estimated from blood oxygenation level dependent (BOLD) images, edges represent the correlation in activation between pairs of regions, as neuronal firing is shortly followed by a depletion of oxygen in surrounding capillaries as neurons prepare to fire again.

The process of estimating a connectome from MRI data involves multiple steps, each with different inputs and outputs, and can be performed with a variety of parameters. There are two main approaches that can be taken in the designing of a pipeline for estimating connectomes: (1) optimize the pipeline for each data set you wish to analyze or (2) design a "general" pipeline that can be used on a variety of data. While the creating of a optimized pipeline for each data set results in the "most accurate" connectomes for the data, it also limits the ability for the pipeline to be used with new data and for comparisons between data sets. Inversely, the creation of a "standard" pipeline which can adequately estimate connectomes from a wide collection of data sets will allow for more confident comparison at the expense of potentially sub-optimal connectome estimation. While there exist multiple pipelines which perform part or all of the processes required to estimate a connectome from MRI data, few exist which are easily accessible for individuals new to the field. Both fmriprep and dmriprep do not estimate a connectome from provided data, rather serving to preprocess data for further analysis by 


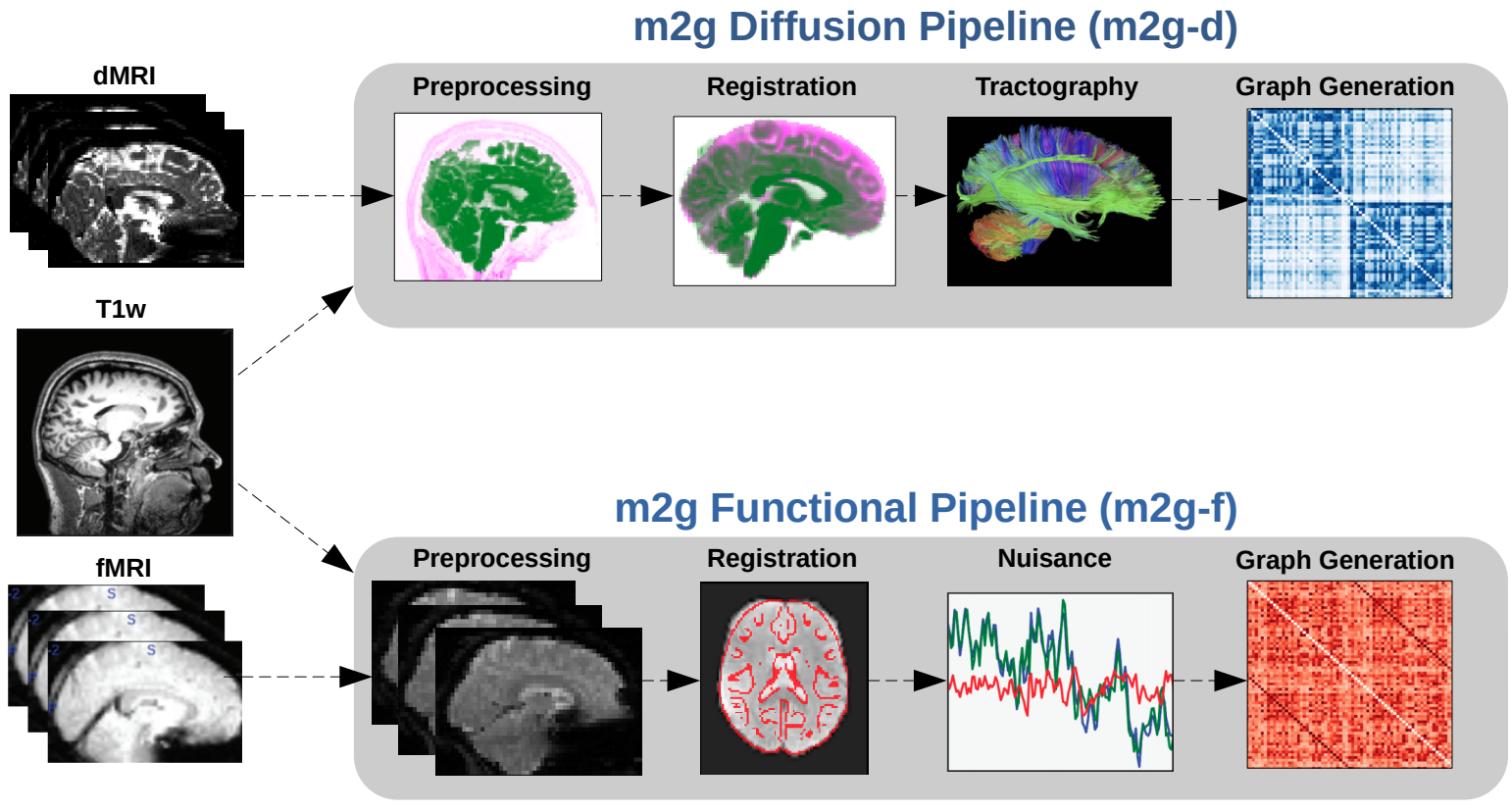

Figure 1: Individual Level Pipeline The $\mathrm{m} 2 \mathrm{~g}$ pipeline has two sub-pipelines: $\mathrm{m} 2 \mathrm{~g}-\mathrm{d}$ transforms Nifti-formatted dMRI data into sparse structural connectomes, and $\mathrm{m} 2 \mathrm{~g}-\mathrm{f}$ organizes the data for processing by CPAC's functional pipeline that we developed here. Each sub-pipeline consists of four key steps, and each step generates both data derivatives and quality assurance figures to enable both qualitative assessments and quantitative comparisons.

other software. Other MRI connectome estimating pipelines often require multiple stages of installation and require significant background knowledge in order to utilize effectively. The Human Connectome Pipeline (HCP) requires the manual installation of multiple software packages and running of different shell scripts for each step of the pipeline, requiring the user to input settings which require extensive knowledge.:

In hopes to expand the study of connectomes to a larger group of individuals, we developed a pipeline, called "MRI to Graphs" (m2g), which utilizes accepted approaches for connectome generation along with multiple open-source tools. The $\mathrm{m} 2 \mathrm{~g}$ pipeline serves to streamline the process of estimating connectomes for both functional and structural MRI data through handling the required preprocessing, graph generation, and quality assurance from one function call. Rather than providing bespoke analyses for each unique dataset, limiting their generalizability and likely increasing computational cost, $\mathrm{m} 2 \mathrm{~g}$ harmonizes processing to produce maximally reliable data derived quantities across a wide variety of datasets. To further the goal of a pipeline accessible to everyone, $\mathrm{m} 2 \mathrm{~g}$ also provides extensive quality assurance at each data processing stage in the form of easy to understand images, using widely accepted visualization formats.

In total, $\mathrm{m} 2 \mathrm{~g}$ processed 13 diffusion-weighted MRI (dMRI) studies comprising $\approx 720$ individuals with $\approx 1,400$ scans, and 30 functional MRI (fMRI) studies comprising $\approx 1,400$ individuals with $\approx 3,500$ scans-yielding a total of approximately 172,000 estimated structural and functional connectomes from all 35 parcellations. Estimation of each of these connectomes was performed with one command line call, 3 CPUs, and $\approx 16$ GB of RAM, in under an hour and a half from raw data to connectome. These connectomes, in addition to code and other data derivatives, are publicly available at https: //neurodata.io/mri/.

\section{Results}


2.1 The $\mathrm{m} 2 \mathrm{~g}$ Pipeline The $\mathrm{m} 2 \mathrm{~g}$ pipeline consists of two separate sub-pipelines: $\mathrm{m} 2 \mathrm{~g}-\mathrm{d}$, which processes diffusion-weighted MRI scans in order to estimate structural connectomes, and $m 2 g-f$, which processes BOLD functional MRI scans in order to estimate functional connectomes. While $\mathrm{m} 2 \mathrm{~g}-\mathrm{d}$ sub-pipeline is locally contained in $\mathrm{m} 2 \mathrm{~g}, \mathrm{~m} 2 \mathrm{~g}-\mathrm{f}$ sub-pipeline has been added into CPAC as a Nipype pipeline and that implementation is called during the processing of functional MRI data. While the parameters and functions were developed and chosen by our group, and the performance is monitored for consistency between updates, the $\mathrm{m} 2 \mathrm{~g}-\mathrm{f}$ sub-pipeline itself is hosted and maintained by the FCP-INDI organization. The steps involved in each of these sub-pipelines are further discussed in the Methods section. Due to the focus on ease of use and generalizability, $\mathrm{m} 2 \mathrm{~g}$ only requires the path of the input directory, output directory, and which of pipelines is to be run. Through calling $\mathrm{m} 2 \mathrm{~g}$ with the simplest parameters:

$$
\text { m2g --<type> <input_directory> <output_directory> }
$$

where <type> is either dwi for the diffusion pipeline or func for the functional pipeline, $\mathrm{m} 2 \mathrm{~g}$ is able to estimate connectomes. The $\mathrm{m} 2 \mathrm{~g}$ pipeline was used to successfully estimate all of the connectomes used in this manuscript. The data consisted of both functional and diffusion MRI nifti files of varying voxel resolutions and acquisition methods. $\mathrm{m} 2 \mathrm{~g}$ is also able to process scans with non-isotropic voxels without issue. This is due to the reslicing preformed in both the $\mathrm{m} 2 \mathrm{~g}-\mathrm{d}$ and $\mathrm{m} 2 \mathrm{~g}-\mathrm{f}$ pipelines, utilizing Dipy's reslice function with tri-linear interpolation for both [2]. As reslicing an image to a different resolution results in the creation of new voxels with different intensity values, it may cause concern to significant changing of the information contained within the images and by extension the connectomes. However, it has been shown that such processing does not significantly change the information for both diffusion and functional MRI [3, 4].

In addition to visual inspection of quality assurance (QA) figures generated after each step in the pipeline, the discriminability metric, which evaluates the fraction of measurements from the same individual that are closer to one another than they are to the measurement of any other individual [5], was used as a benchmark to the performance of $\mathrm{m} 2 \mathrm{~g}$ during development. The $\mathrm{m} 2 \mathrm{~g}-\mathrm{d}$ pipeline was optimized on the Kirby21 dataset [6], while the $\mathrm{m} 2 \mathrm{~g}-\mathrm{f}$ pipeline was optimized on both the Kirby21 and IBATRTdatasets because of their creation for test-retest analysis Both pipelines were then validated using data from the Consortium of Reliability and Reproducibility (CoRR), consisting of 35 different studies from nearly 20 different institutions around the world, spanning the Americas, Europe, and Asia [7]. The CoRR data collection efforts were not harmonized, and all data (regardless of quality) were requested to be shared. This data repository was thus well-suited to test the robustness of our pipeline. Several additional open access datasets from other repositories with different acquisition details, such as ABIDE [8, 9], were also processed with $\mathrm{m} 2 \mathrm{~g}$.

The neuroparc repository of atlases, developed alongside $\mathrm{m} 2 \mathrm{~g}$ was used as the main resource for the standardized parcellations used in m2g's development [10]. This repository served to consolidate atlases from a multitude of sources, each registered to MNI152 space at 1, 2, and $4 \mathrm{~mm}^{3}$ voxel sizes. Of the atlases listed there, 35 were used during the development of $\mathrm{m} 2 \mathrm{~g}$. While neuroparc was developed in unison with $\mathrm{m} 2 \mathrm{~g}$, the $\mathrm{m} 2 \mathrm{~g}$ pipeline is capable of utilizing unique parcellation atlases provided by the user.

Due to the lack of gold standards to determine the accuracy of an estimated connectome, certain forms of validation of the $\mathrm{m} 2 \mathrm{~g}$ pipeline are impossible. However, we have used surrogate metrics for $\mathrm{QA}$ to increase confidence in the outputs. For each MRI, $\mathrm{m} 2 \mathrm{~g}$ ran to completion while passing basic QA metric consisting of (1) edge values associated with every ROI, (2) for all applicable datasets, discriminability values above 0.7 , (3) QA figures generated successfully, (4) homophyly and homotopy observations in agreement with literature. $\mathrm{m} 2 \mathrm{~g}$ 's ability to pass these metrics for validation denote its ability to be used widely, across a variety of datasets, including those leveraging older MRI technology. The resulting connectomes constitute one of the larger open database of connectomes to date.

To visually inspect consistency in connectomes across the datasets, a distance-dependent group 
consensus structural connectivity graph was created. This method serves to prevent the observed trend of down-weighting long range connections [11] in conventional averaging of connectomes and preserve the network properties/hubb-ness seen in individuals. A similar graph was created for the functional MRI data, called a group-averaged functional connectivity matrix [12], which serves to minimize the effect of inconsistent correlations (Figure 2). The reproducibility of the estimated connectomes was tested using discriminability [5], and network statistics to determine that biological diversity and plausibility were maintained.

2.2 Validated Connectomes Connectomes were estimated by both $\mathrm{m} 2 \mathrm{~g}-\mathrm{d}$ and $\mathrm{m} 2 \mathrm{~g}-\mathrm{f}$ for all 35 parcellations from the Neuroparc repository [10] As mentioned before, the oracle accuracy of connectomes compared to true functional and structural properties of individuals' brains is impossible to measure. Instead, we measure the discriminability of each dataset (Figure 3), which is a measure of preservation of individual-specific information and data repeatability. We also observed trends in agreement with existing literature regarding the edge strengths between regions of interest and relative amount of homophyly and homotopy (Figure 4). Manual inspection of the extensive quality control checks associated with each step, particularly those regarding registration, served to reinforce our confidence in the generated connectomes.

Discriminability Discriminability was estimated for each data set, for every data set with multiple scans per subject (as reported in the Supporting Materials, which gives all of the individual numeric values). The closer to 1 the discriminability value, the more distinct the connectomes generated from one individual's scans are from the connectomes of others. The $\mathrm{m} 2 \mathrm{~g}-\mathrm{d}$ pipeline created connectomes with discriminability values greater than 0.7 for the 33 of the 35 parcellations (Figure 3, top). The discriminability measurements appear to be robust to the number of scans per subject, as can be seen with the SWU4 and HNU1 data sets (two scans per subject and ten scans per subject, respectively). In addition, $\mathrm{m} 2 \mathrm{~g}-\mathrm{f}$ relatively rarely created connectomes with discriminability values lower than 0.7 (Figure 3 , bottom).

Connectome Connectivity Because discriminability assesses the uniqueness of each individual's connectome, it does not guarantee that the connectome itself is biologically meaningful. To address this issue, we analyzed the prominence of different categories of connections-ipsilateral versus contralateral and homotopic versus heterotopic-using the connectomes estimated from three unique parcellations. To analyze homotopic connections, we chose the DKT [13], AAL [18], and Hammersmith [19] parcellations, due to their symmetric ROI placement (i.e. each ROI on the left hemisphere had a matching $\mathrm{ROI}$ on the right). For the structural connectomes, the percentage of total edges which belonged to each network were analyzed, while the the Pearson's correlation value [20] calculated by CPAC was used for the functional connectomes. The results coincided with well documented phenomena regarding structural and functional relationships [14-17]. Namely, structural connectomes had significantly more ipsilateral than contralateral connections than homotopic, while functional connectomes had significantly stronger homotropic correlations than heterotropic either ipsilaterally or contralaterally (Figure 4).

Reproducibility In the $\mathrm{m} 2 \mathrm{~g}$ pipeline, there exists algorithms with the potential to introduce noise in the generated connectome. The randomized seed placement in the $\mathrm{m} 2 \mathrm{~g}-\mathrm{d}$ pipeline, and the registration of the functional MRI data onto the T1-weighted image and the T1-weighted image onto the diffusion MRI data for $\mathrm{m} 2 \mathrm{~g}-\mathrm{f}$ and $\mathrm{m} 2 \mathrm{~g}-\mathrm{d}$, respectively, serve as potential vectors for noise. To test the reproduciblity of $\mathrm{m} 2 \mathrm{~g}$, a subset of scans were used to estimate connectomes multiple times. Using a subset of five scans from each dataset, $\mathrm{m} 2 \mathrm{~g}$ estimated connectomes five times per scan, using the same default parameters. The resulting connectomes were treated as unique connectomes, with each set belonging to the same subject. Discriminability metrics were calculated for each of these repeated connectomes, to determine whether the unique qualities of the individual scan were preserved. For all subsets, the discriminability 
bioRxiv preprint doi: https://doi.org/10.1101/2021.11.01.466686; this version posted November 3, 2021. The copyright holder for this preprint (which was not certified by peer review) is the author/funder, who has granted bioRxiv a license to display the preprint in perpetuity. It is made available under aCC-BY-NC-ND 4.0 International license.
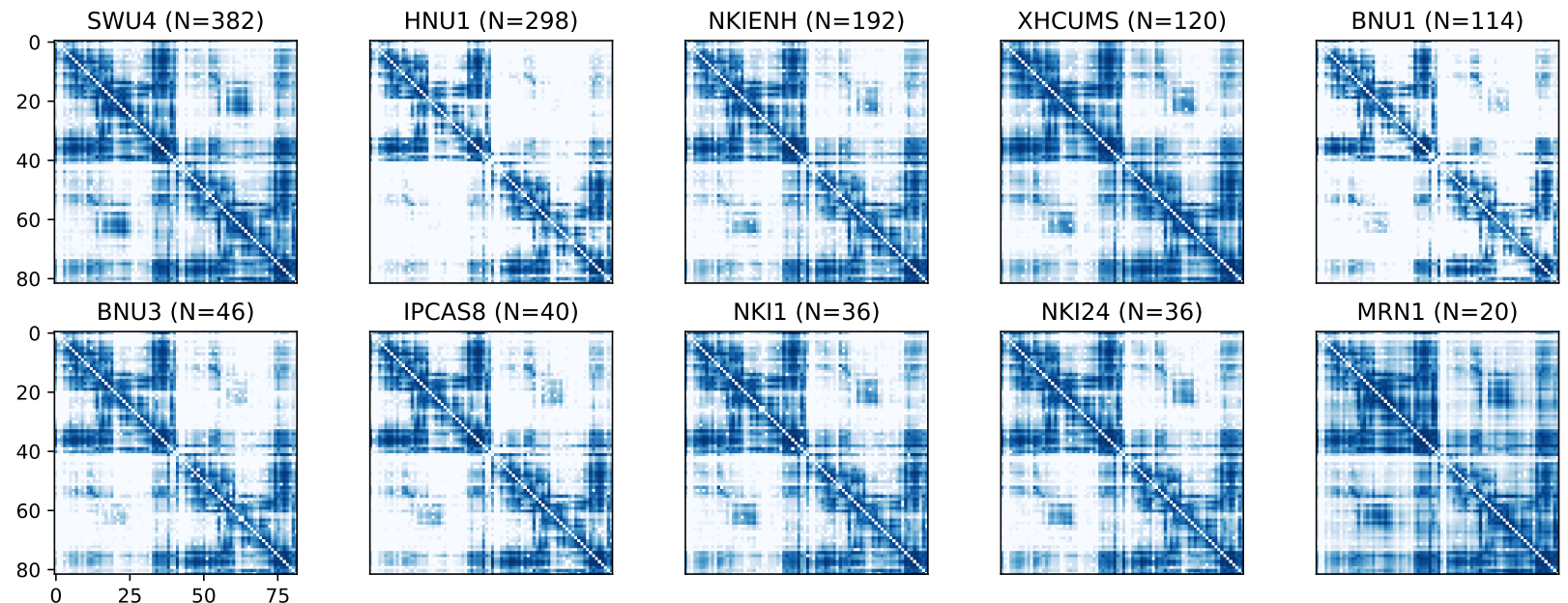

MRN1 $(\mathrm{N}=20)$
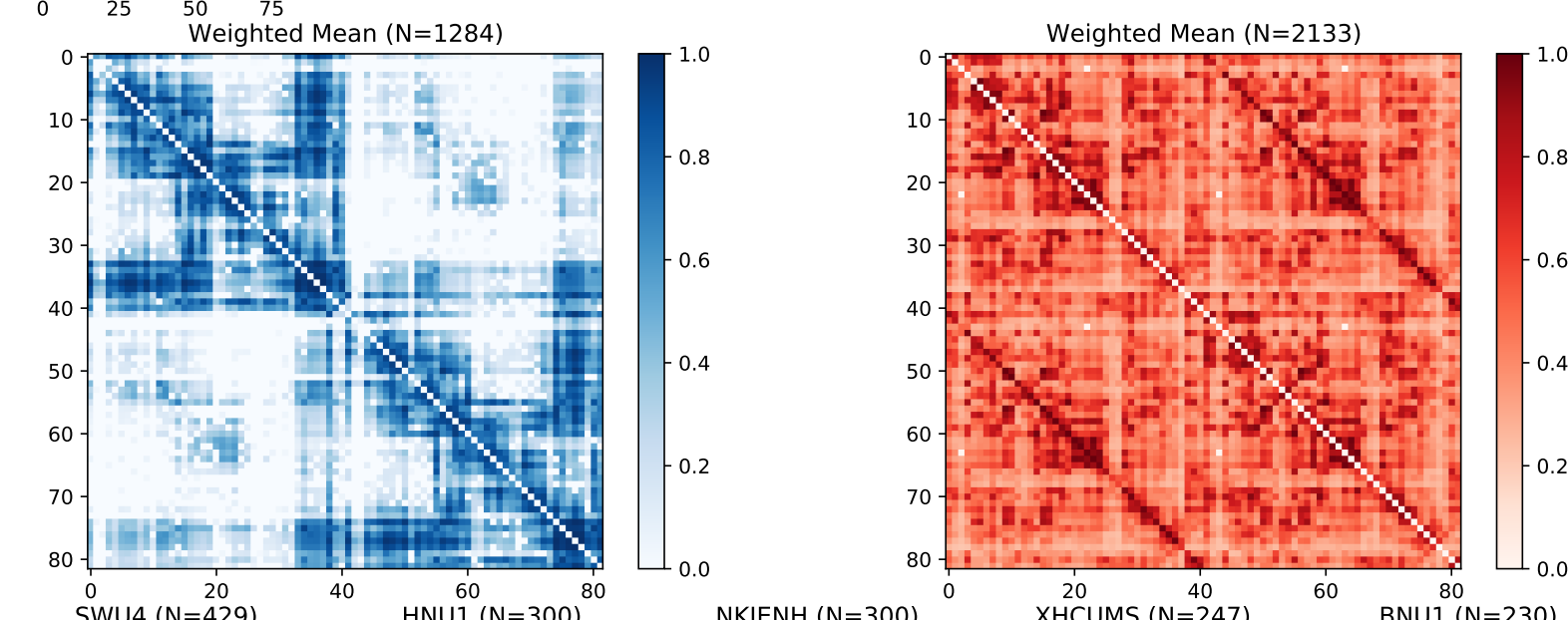

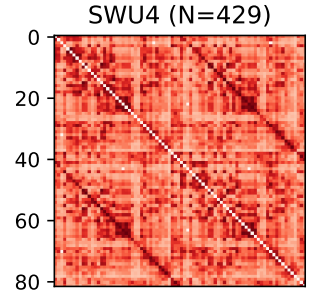

BNU3 $(\mathrm{N}=144)$

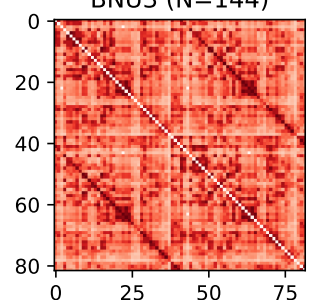

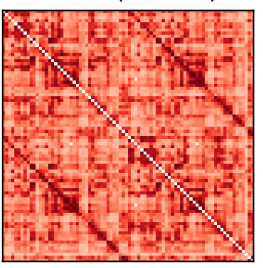

IPCAS8 $(\mathrm{N}=144)$

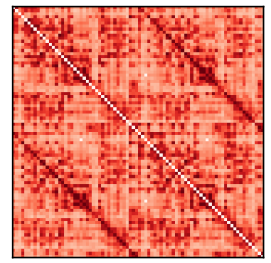

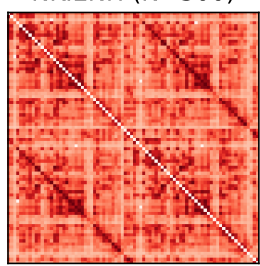

NKI1 $(\mathrm{N}=119)$

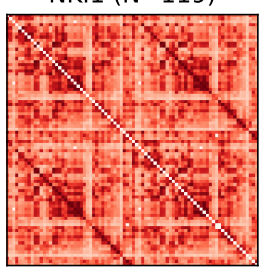

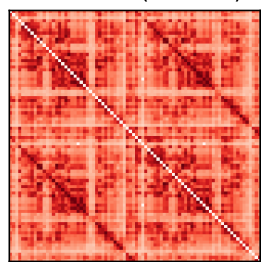

NKI24 $(\mathrm{N}=114)$

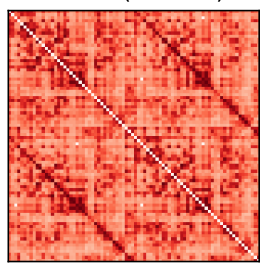

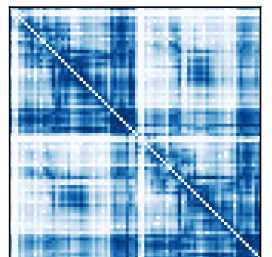

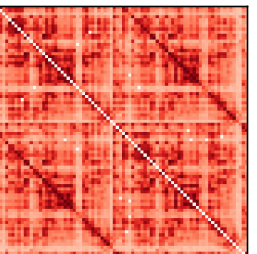

MRN1 $(\mathrm{N}=106)$

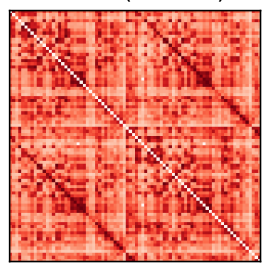

Figure 2: Group Consensus Connectomes Group consensus structural connectome from $\mathrm{m} 2 \mathrm{~g}-\mathrm{d}$ (blue) and group consensus functional connectomes from $\mathrm{m} 2 \mathrm{~g}-\mathrm{f}(\mathrm{red})$, using the DKT parcellation method [13]. Structural connectomes of the datasets appear qualitatively similar, with minor deviations particularly visible in the contralateral regions of the connectomes (nodes $0-40$ and 41-80). Ipsilateral connectivity is consistently more dense than contralateral connectivity in structural connectomes [14]. The functional connectomes appear qualitatively similar to one another. Homotopic correlation is consistently higher than ipsilateral and contralateral connectivity, which agrees with existing knowledge about functional correlation in the brain [15-17]. 
bioRxiv preprint doi: https://doi.org/10.1101/2021.11.01.466686; this version posted November 3, 2021. The copyright holder for this preprint (which was not certified by peer review) is the author/funder, who has granted bioRxiv a license to display the preprint in perpetuity. It is made available under aCC-BY-NC-ND 4.0 International license.
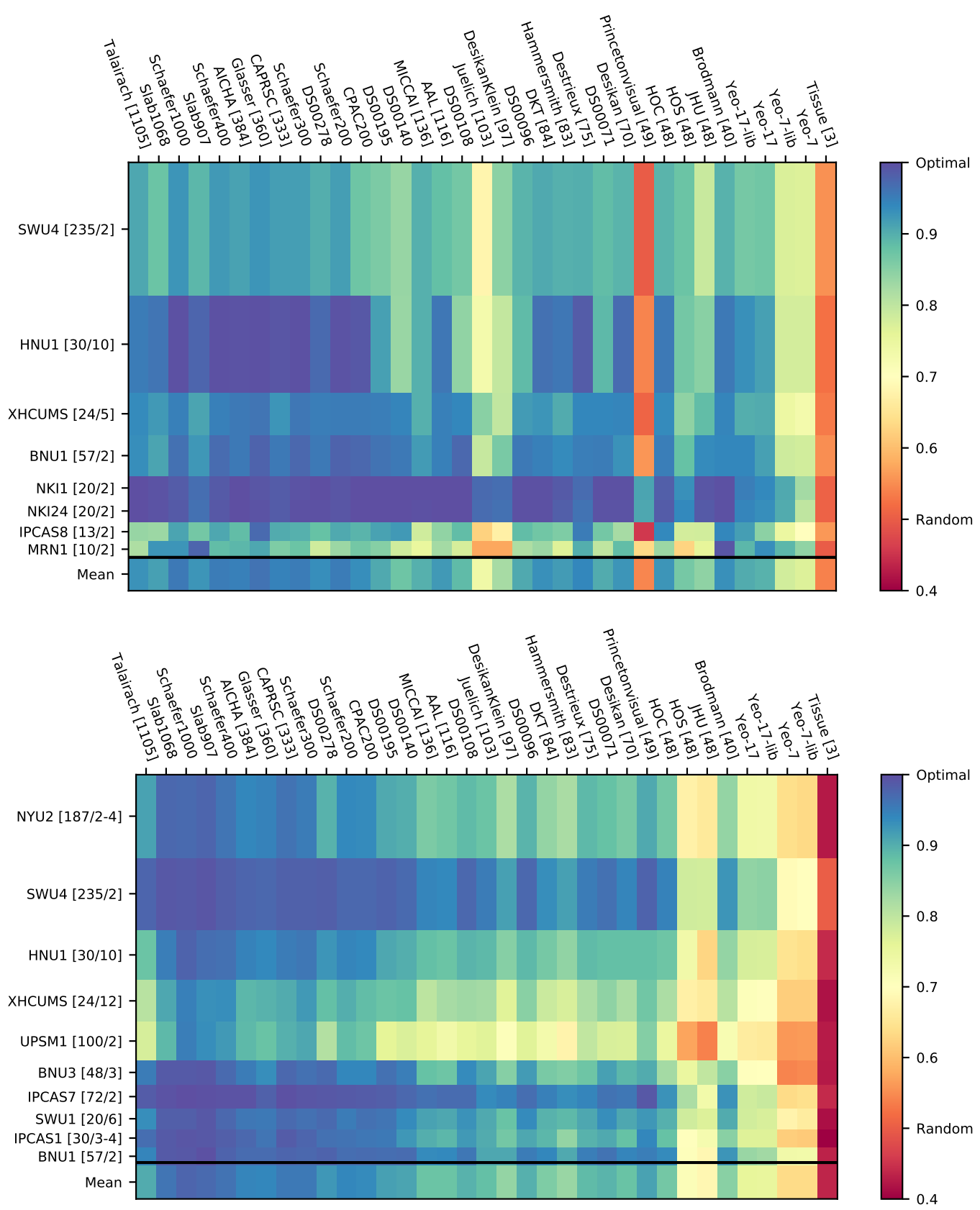

Figure 3: Discriminability Results Discriminability values from each applicable diffusion (top) and functional (bottom) MRI dataset. Relative row height denotes the relative size of the dataset. Columns, each representing a different parcellation, are organized from left to right by highest to lowest number of ROls. The mean discriminability value for each parcellation is displayed in the last row of both plots. The number of subjects/sessions is displayed next to the datasets' names in brackets and the number of ROl's in a given parcellation are shown in brackets if not mentioned in the parcellation name. Discriminability values for the structural connectomes was greater than 0.7 for the 32 of the 35 parcellations, while being robust to the number of scans per subject. Functional connectomes rarely had discriminability values lower than 0.7 .

values greater than 0.97 were recorded for all parcellations (excluding the Tissue parcellation method) for both the functional and structural connectomes. Additional testing using Spearman's rank correlation found that the repeated connectomes had a coefficient greater than 0.98 for all parcellations.

2.3 Low Resource and Time Requirement Computation expediency and resource efficiency were key reasons to develop $\mathrm{m} 2 \mathrm{~g}$. While the required resources for running $\mathrm{m} 2 \mathrm{~g}$ varies according to the data, 


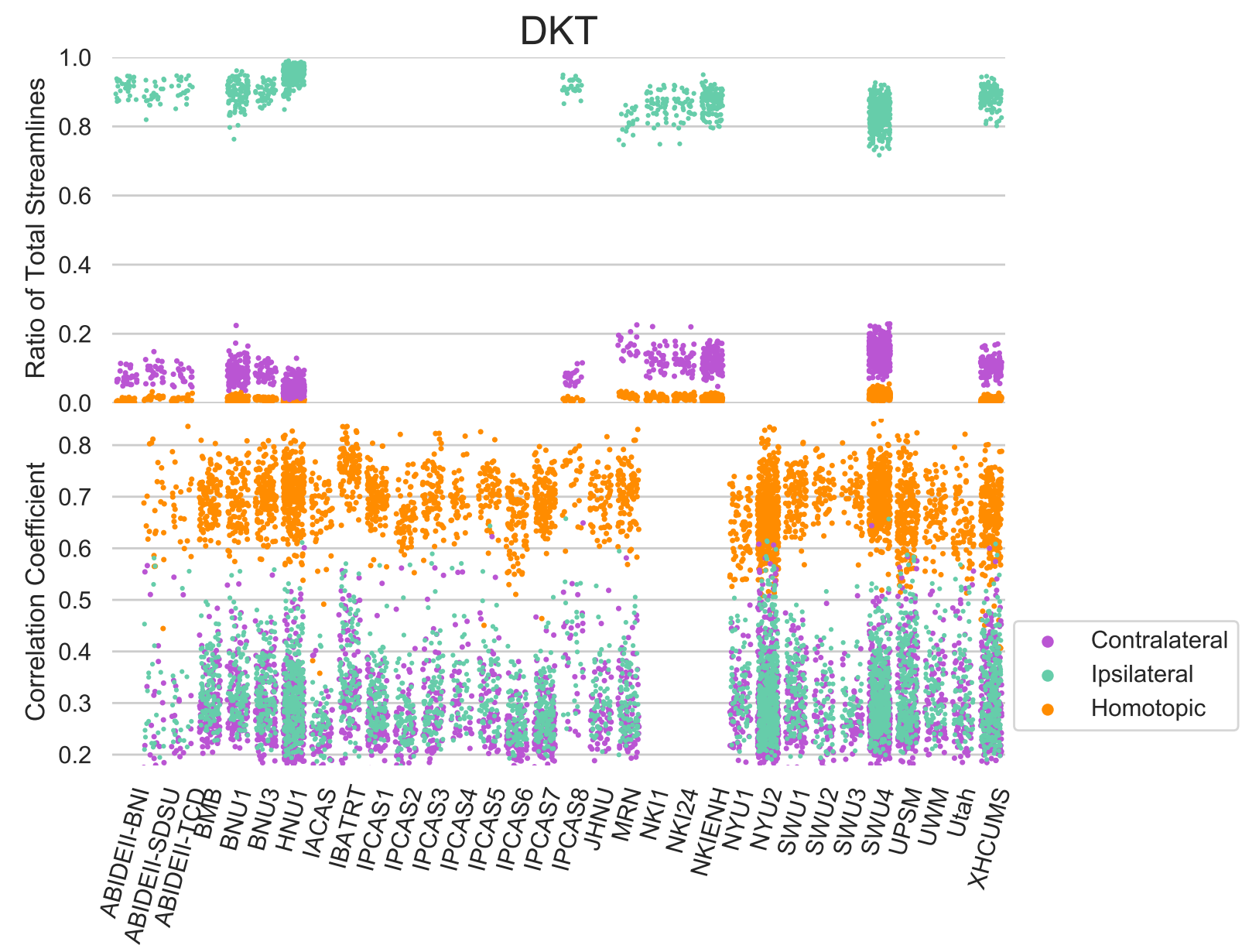

Figure 4: Connectome biological plausibility results Analysis of the edge weights of both structural (top) and functional (bottom) connectomes estimated using the DKT, AAL, and Hammersmith parcellation methods. For the structural connectomes, the mean of the percentage of streamlines observed between ipsilateral, contralateral, and homotopic ROls was recorded and plotted for each scan. Mean Pearson correlation coefficients between ipsilateral, contralateral, and homotopic ROls was plotted for functional connectomes. A consistent significantly higher ratio of ipsilateral connections was observed across parcellation methods for structural connectomes, as well as a higher correlation between homotopic ROls across parcellation methods in functional connectomes.

the datasets mentioned in this paper could be processed by $\mathrm{m} 2 \mathrm{~g}$ with approximately $16 \mathrm{~GB}$ of RAM and $1 \mathrm{CPU}$ core. If more resources are available, $\mathrm{m} 2 \mathrm{~g}$ is capable of utilizing multiple CPUs at the same at various points throughout the pipeline, such as registration and graph generation, to reduce runtime. While estimating the connectomes used in this manuscript, we recorded the time required for $\mathrm{m} 2 \mathrm{~g}$ to successfully analyze each of the scans used in the discriminability calculations (Figure 5). The optimal configuration of resources for our purposes required $3 \mathrm{CPUs}$ and $16 \mathrm{~GB}$ of RAM. When tested using 1 $\mathrm{CPU}, \mathrm{m} 2 \mathrm{~g}-\mathrm{d}$ took less than 120 minutes to complete the connectome creation using 35 parcellations for a given dMRI scan. The $\mathrm{m} 2 \mathrm{~g}-\mathrm{f}$ pipeline likewise took less than 110 minutes to generate the set of connectomes. Additional CPUs were found to significantly increase the RAM requirements of the $\mathrm{m} 2 \mathrm{~g}-\mathrm{f}$ pipeline, due to the configuration of CPAC.

2.4 Quality Assurance Outputs Quality assurance images were created by $\mathrm{m} 2 \mathrm{~g}$ at each of the significant steps of both the $m 2 g-d$ and $m 2 g-f$ pipelines (Figure 1 ) for easy determination of erroneous results. The QA figure formats were chosen for maximum clarity and minimal image analysis expertise requirements in order to detect any potential errors. Documentation for each of the QA figures generated by $\mathrm{m} 2 \mathrm{~g}$ can be found at https://neurodata.io/mri/. 

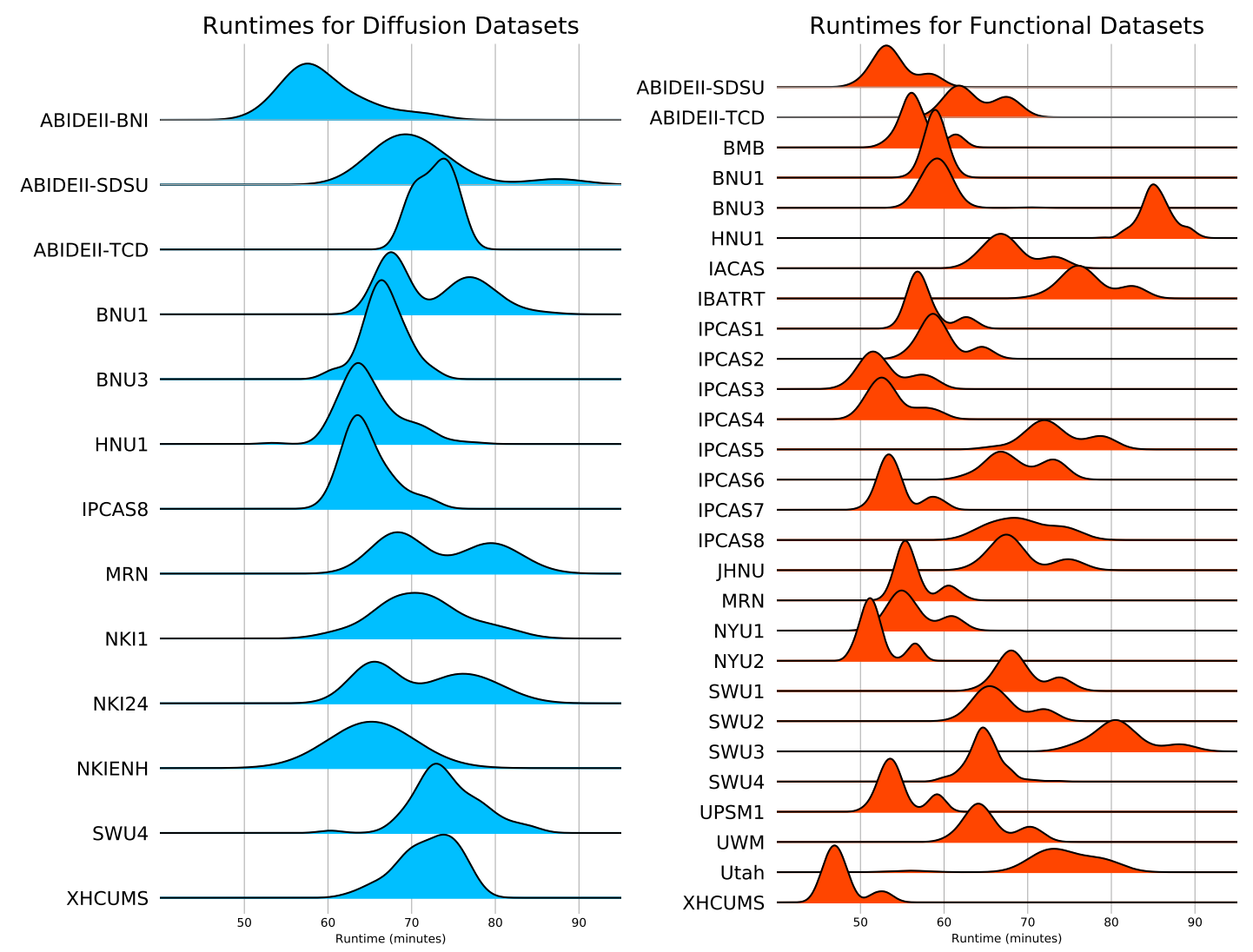

Figure 5: Runtimes for Diffusion and Functional Datasets Amount of time, in minutes, it took for $\mathrm{m} 2 \mathrm{~g}$ to generate a connectome for each of the scans in the datasets using the 35 brain parcellations. Computation time for diffusion datasets (blue) and functional datasets (red) varied based off of size and resolution of the input MRI files. In these time estimates, $\mathrm{m} 2 \mathrm{~g}$ was run utilizing 3 CPUs in parallel and at least 16 GB of RAM available.

3 Discussion The $\mathrm{m} 2 \mathrm{~g}$ processing pipeline was created to both efficiently analyze diffusion and functional MRI scans, and lower the barrier for entry to connectomics. It was developed to excel at three key aspects: ease of use, biological veracity, and computational reproducibility. With regards to these three aspects, we believe $\mathrm{m} 2 \mathrm{~g}$ to be a success.

Attesting to its ease of use, all datasets referenced in this manuscript were run through $\mathrm{m} 2 \mathrm{~g}$ with the default parameters and minimal user input, resulting in the successful generation of connectomes for each scan. This was true across a range of acquisition properties of the MR images. Moreover, this processing was achieved with modest computational resources, 16 GB of RAM and $1 \mathrm{CPU}$ and no intra-pipeline additional parallel processing steps, in less than two hours for each scan. In addition, $\mathrm{m} 2 \mathrm{~g}$ requires very minimal user input, only the filenames, voxel size, and acquisition method (for fMRI data). As such, the pipeline is an easy tool for researchers and clinicians without extensive computer science experience or resources. Finally, $\mathrm{m} 2 \mathrm{~g}$ produces comprehensive visual reports to analyze processing output.

We assessed m2g's ability to produce biologically plausible and realistic data using multiple approaches. First, we computed the discriminability for each dataset. Discriminability scores depend on the parcellation method used in the connectome generation. However, the discriminability values surpassed statistical tests of random chance in essentially all cases. The majority of the 35 parcellation methods used in this analysis resulted in discriminability values above 0.8 (Figure 3). Upon re-running of datasets though the $\mathrm{m} 2 \mathrm{~g}$ pipeline, these discriminability values changed by less than 0.03 for the HNU1, SWU4, and BNU1 datasets. We also were able to confirm the negligible effect variance in connectome generation introduced by randomized elements of the pipeline. 
Second, we assessed the relative fraction of ipsilateral versus contralateral, and homotopic versus heterotopic, connections. Results were consistent with current knowledge about the anatomical and functional realities of neuro-typical brains [14-17]. More specifically, the prominence of ipsilateral connections in structural connectomes, and the prominence of homotopic correlations in functional connectomes (Figure 4).

Third, our focus on computational reproducibility manifested through the generation of QA figures for every major step in both the $\mathrm{m} 2 \mathrm{~g}-\mathrm{d}$ and $\mathrm{m} 2 \mathrm{~g}-\mathrm{f}$ pipelines. With users without extensive knowledge of MRI analysis in mind, the QA figures created by $\mathrm{m} 2 \mathrm{~g}$ were designed to make it easy to visually determine whether the pipeline has successfully processed their data. With the emphasis on color coordination and concise figure generation, any issue in the preprocessing and registration process is made obvious. All QA figures are clearly labeled and placed in the same directory location relative to the output, regardless of pipeline being used, with detailed explanations found at http://m2g.io.

The design criteria for $\mathrm{m} 2 \mathrm{~g}$ required certain trade-offs in performance to increase the generalizability of input data. With the strength of pipeline flexibility and minimal required user input still being paramount, $\mathrm{m} 2 \mathrm{~g}$ could be improved along several dimensions. First, recent advances in registration [21] and tractography [22] could be incorporated. Second, several more sophisticated batch effect strategies have been successfully employed in dMRI data [23]. Such strategies could possibly help here as well, especially if they are modified appropriately to work on binary graphs [24]. Third, $\mathrm{m} 2 \mathrm{~g}$ may underperform for particular populations (e.g., infants) or for brains that show nonstandard structures such as tumors, resected regions, or lesions. The refinement of the pipeline used adult brains, with no current measurement on how it would react to significantly different scans. Inclusion of the ability to supplement the standard MNI152 reference images and parcellations with ones relevant to nonstandard brain structures could drastically expand the populations $\mathrm{m} 2 \mathrm{~g}$ could process. This would be particularly interesting as a means to adapt the workflow to data collected from rodents and nonhuman primates in the future.

Because the methods developed during the creation of $\mathrm{m} 2 \mathrm{~g}$ are open source and easy to use, and the data are open access, the continual development of the pipeline and assessment of the connectomes generated is ready for outside collaboration. Due to the well-commented and modular code, modification is straightforward for pythonistas. Along with the ease of use, a boon for the wider adoption of $\mathrm{m} 2 \mathrm{~g}$ is the plethora of connectomes created as a byproduct of its development. With each of the MRI datasets being processed on 35 unique parcellation methods, possibly the largest collection of connectomes has been created. These connectomes are open access and available from our website, https://neurodata.io/mri/.

\section{Methods}

4.1 Data The $\mathrm{m} 2 \mathrm{~g}$ pipelines were validated through the processing of the majority of data from the Consortium of Reliability and Reproducibility (CoRR). The CoRR data consists of 36 different studies from nearly 20 different institutions around the world, spanning the Americas, Europe, and Asia [7]. The CoRR data collection efforts were not harmonized, and all data (regardless of quality) were shared. Thus, these collections are well-suited to test the robustness of any pipeline. In addition to the CoRR data, we also used $\mathrm{m} 2 \mathrm{~g}$ to process several additional open access data collections with complementary acquisition parameters. Using this collection of MRI data, $\mathrm{m} 2 \mathrm{~g}$ pipeline development and parameter selection prioritized the maximization of discriminability scores across all datasets, while also focusing on limiting resource requirements.

$4.2 \mathrm{~m} 2 \mathrm{~g}$ Input The input required to run the $\mathrm{m} 2 \mathrm{~g}$ pipeline depends on whether functional and/or diffusion MRI data is being analyzed. The required inputs for either pipeline are:

- A T1-weighted anatomical scan, either an uncompressed or gzipped nifti file

- $\mathrm{m} 2 \mathrm{~g}-\mathrm{d}$ :

- A diffusion-weighted MRI file, either an uncompressed or gzipped nifti file 
- b-value and b-vector parameter files (.bval and .bvec file types)

- A functional MRI file, either an uncompressed or gzipped nifti file

- The TR value (in seconds) and acquisition method used for the functional scan

The directory containing the input data must be BIDS formatted [25]. Both the $\mathrm{m} 2 \mathrm{~g}-\mathrm{d}$ and $\mathrm{m} 2 \mathrm{~g}-\mathrm{f}$ pipelines can be run independently or on the same dataset, provided the required data files are available. Which parcellation(s) to be used in the connectome creation can be specified by the user, whether they be unique files stored locally or from the neuroparc repository [10]. If no parcellations are present, then $\mathrm{m} 2 \mathrm{~g}$ will pull from the neuroparc repository and run all available parcellations.

$4.3 \mathrm{~m} 2 \mathrm{~g}$ Diffusion Workflow The $\mathrm{m} 2 \mathrm{~g}$ diffusion pipeline leverages existing open source tools, including the fMRI Software Library (FSL) [26-28], Dipy [2], the MNI152 atlas [29], and a variety of parcellations defined in the MNI152 space [10]. The pipeline consists of five major steps: (1) Preprocessing, (2) Registration, (3) Tensor estimation, (4) Tractography, and (5) Graph generation. All algorithms used throughout the pipeline requiring hyper-parameter selection were initially set to the suggested parameters, and optimized on the subset of CoRR datasets. The output of each processing stage includes data derivatives and QA figures to enable individualized accuracy assessments. The QA figures at each major step include cross-sectional images at different depths in the three canonical planes (sagittal, coronal, and axial) of images or overlays. Figure 1 provides a schematic of the individual-level analysis.

Preprocessing The input diffusion MRI data is first eddy-corrected using FSL's eddy_correct [2] program with the default parameters. This program was chosen as newer eddy functions either require substantially longer to run or rely on GPU acceleration, an additional resource requirement which would reduce the accessibility of $\mathrm{m} 2 \mathrm{~g}$. The associated bvec and bval files are then checked for any errors and, if necessary, corrected using Dipy's read_bvals_bvecs function [2] into a usable format. The b-vectors are then normalized, making sure to not alter the b-vector if its associated b-value is 0 .

The eddy-corrected dMRI file then has its orientation checked, and if not already oriented in RAS+ coordinate space, nibabel's as_closest_canonical [30] is used for the reorientation. The b-vectors are also reoriented to RAS+ if necessary using default parameters. The dMRI file is then resliced to the desired voxel size (specified by the user, with a reasonable default of $2 \mathrm{~mm}^{3}$ if not specified) using Dipy's reslice function with trilinear interpolation[2].

The T1 weighted MRI file (T1w) is also reoriented into RAS + format using as_closest_canonical and similarly resliced, if necessary, into the desired voxel size using Dipy's reslice function [2] with default parameters. The resulting image is then skull-stripped using AFNI's 3dSkullStrip function [31] with the surface density parameter set to 30 , resulting in an anatomical image with all non-neuronal structures removed.

Registration From the skull-stripped T1w anatomical file, three probability masks are generated for the grey matter, white matter, and cerebral spinal fluid regions of the brain using FSL's fast function [26-28] using default parameters. The white matter mask is then used to create another mask of just the outer edge of the white matter area using FSL's fslmaths [26-28]. Using FSL's flirt function [26-28], an initial linear registration is made for the anatomical image to the reference MNI152 template in MNI space. An initial attempt is made for non-linear registration of the T1w image to the MNI template, using the affine transformation matrix previously created by the linear registration as a starting configuration. If it is not successful and throws an error, the linear registration is used. Using FSL's fnirt function [26-28], the nonlinear warp coefficients/field is generated to register the T1w image onto the reference MNI image. FSL's invwarp function [26-28] is then used on the coefficients/field to get the field for registering the reference $\mathrm{MNI}$ image onto the T1w image. However, if the nonlinear registration fails, then the linear registration affine transformation matrix for $\mathrm{T} 1 \mathrm{w}$ to $\mathrm{MNI}$ is used and inverted using FSL's convert_xfm 
[26-28].

FSL's flirt is then used for calculating the boundary-based registration (BBR) of the dwi image onto the T1w image, using 256 bins and 7 degrees of freedom. The resulting transformation matrix is inverted and used with a mutual information cost function in flirt to generate an image of the anatomical image registered to the dwi image. Using fsImaths, binary ventricle and corpus callosum masks in MNI space are generated. Along with the previously generated grey matter, white matter, and cerebral spinal fluid masks, flirt is used to register the masks to dwi space. A grey matter/white matter boundary binary mask is then made using fslmaths, which denotes the approximated boundary between the two tissue types across the entire brain, for later seed propagation in the tractography stage of the pipeline.

Finally, the parcellation files to be used in the graph generation are reoriented using nibabel's as_closest_canonical function into RAS+, where they are resliced to the desired voxel size using Dipy's reslice function. They are then registered from $\mathrm{MNI}$ to dwi space using the previously calculated transformation matrices, utilizing FSL's flirt function and the "nearest neighbor" cost function.

Tractography Seeds are generated along the grey matter/white matter boundary mask of the registered brain using a custom python script, where the number of seeds per voxel on the mask can be specified by the user. The seed locations and dwi image are then used in conjunction with Dipy tractography functions [2] in order to generate a series of non-directional streamlines spanning the image. $\mathrm{m} 2 \mathrm{~g}$ offers deterministic or probabilistic tractography, Constrained Spherical Deconvolution (CSD) or Constant Solid Angle (CSA) reconstruction methods, and particle filter or local tracking. Regardless of the tractography settings specified, the resulting series of streamlines is output and saved to a trk file for use in graph generation.

Graph Generation For each parcellation being analyzed, each streamline is converted into voxel coordinates and analyzed for overlap with any of the regions specified by the parcellation. $\mathrm{m} 2 \mathrm{~g}$ does not determine the direction of a given streamline, making its connectome and resulting graph undirected. If a streamline overlaps with a given region for more than $2 \mathrm{~mm}$, it is considered an intersection. The number of streamlines intersecting a given region and the other regions they also intersect with are recorded and tabulated in the form of a weighted edgelist. In this edgelist format each unique region of interest on the parcellation, marked by a unique intensity value, is considered a node whose edge weight is the number of streamlines that connect it to another region. For example, if a streamline intersects two or more regions in the parcellation (region $\mathrm{A}$, region $\mathrm{B}$, and region $\mathrm{C}$ ) the weight of the edges $A B, A C$, and $B C$ is increased by one. In this sense, the order through which the streamline intersects is not important. The resulting edgelist is saved in the form of a csv file, and an accompanying adjacency matrix of the normalized edgelist is also saved. A unique edgelist will be made for each parcellation specified by the user.

$4.4 \mathrm{~m} 2 \mathrm{~g}$ Functional Workflow The $\mathrm{m} 2 \mathrm{~g}-\mathrm{f}$ pipeline was constructed starting with the optimal processing pipeline identified in Wang et. al [5] using CPAC [32]. The CPAC pipeline utilizes existing open source tools, including FSL [26-28], Dipy [2], the MNI152 atlas [29], and a variety of parcellations defined in the MNI152 space [10]. The functional pipeline consists of four major steps: (1) Preprocessing, (2) Registration, (3) Nuisance correction, and (4) Graph generation.

Preprocessing The $\mathrm{m} 2 \mathrm{~g}-\mathrm{f}$ pipeline in CPAC uses AFNI's SkullStripping function [31] with a variable shrink factor between 0.4 and 0.6 over 250 iterations with nearest neighbor interpolation to eliminate all non-neuronal structures from the anatomical image. The resulting anatomical file is resampled to the desired resolution voxel size using FSL's FNIRT [26-28]. Slice timing correction is then performed using AFNI's 3dTshift [31], utilizing the TR value and scan acquisition method provided by the user. The resulting image is then motion corrected using AFNl's 3dvolreg, aligning all images to the first image in the functional MRI's timeseries. 
Registration Nonlinear boundary based registration of the preprocessed $\mathrm{AMRI}$ and anatomical images are performed in order to transform them into MNI152 space. The MNI152 6th generation anatomical reference image [29] is used in this registration process, as it is FSL's preferred image. The functional image is then registered to the anatomical scan using FSL bet [26-28] with BBR. FSL's standard white matter, grey matter, and cerebral spinal fluid masks are then registered onto the functional image using FSL's FAST thresholding, resulting in segmentation tissue masks. The specified atlases that are going to be used in the connectome generation are also registered to the functional space using FSL's FLIRT function.

Nuisance Correction Nuisance correction of the functional image is performed using the component based noise correction (CompCor) method. Using "noise ROls", or areas of white matter and cerebral spinal fluid whose intensity values are unlikely to be modulated by neural activity, physiological noise can be isolated[33]. Based on this assumption, physiological noise in gray matter regions can be corrected for by regressing out principal components from noise ROls. CompCor uses the previously registered white matter and cerebral spinal fluid masks (csf) in order to determine noise ROls. A principal component analysis is applied using the top five components of the white matter and csf to characterize the times series data from the noise ROls. Significant principal components are then introduced as covaraites in a general linear model as and estimate for the physiological noise single space. Polynomial Detrending is also performed to remove linear or quadratic trends in the timeseries, likely from changes in scanner heat or subject movement. After nuisance regression, frequency filtering occurs on the functional data using a bandpass filter from $0.01 \mathrm{~Hz}$ to $0.1 \mathrm{~Hz}$ to account for low-frequency drift and high-frequency noise.

Graph Generation With the anatomical files and atlases registered to the functional image. For each $\mathrm{ROI}$, the timeseries of the average of all voxels within the ROI at each collection time point is calculated. This timeseries is then used to calculate the Pearson's correlation coefficient [20] between each pair of ROls in the given timeseries. The resulting adjacency matrix of correlations is then saved, along with an edgelist file containing the same information.

\subsection{Validation of $\mathrm{m} 2 \mathrm{~g}$ on Diverse Data}

Discriminability To evaluate a methods reliability, Wang et al. [5] developed a metric called discriminability that quantifies the fraction of measurements from the same individual that are closer to one another than they are to the measurement of any other individual. Discriminability, as seen in Equation (4.1), describes that probability that two observations within the same class are more similar to one another than two objects belonging to a different class:

$$
D=p\left(\left\|a_{i j}-a_{i j^{\prime}}\right\| \leq\left\|a_{i j}-a_{i^{\prime} j^{\prime}}\right\|\right)
$$

In the context of validation of $\mathrm{m} 2 \mathrm{~g}$, each connectome is converted into a ranked vector, assigning each edge a weight between 0 and 1 from smallest to largest, and compared. This means that each connectome, $a_{i j}$, in a test-retest dataset is first compared to other connectomes belonging to the same subject, $a_{i j^{\prime}}$, and then to all connectomes belonging to other subjects, $a_{i^{\prime} j^{\prime}}$. A perfect discriminability score is 1 , meaning that for all observations within the dataset, each connectome is more alike to connectomes from the same subject than to others. Optimizing $\mathrm{m} 2 \mathrm{~g}$ with respect to discriminability enables us to minimize the upper-bound on error for any general downstream inference task. The discriminability score for the multitude of datasets run through the default settings of $\mathrm{m} 2 \mathrm{~g}$ were recorded.The average discriminability over all scans was approximately 0.85 for dMRI data and approximately 0.8 for fMRI data.

Connectome Connectivity While discriminability may determine the extent that $\mathrm{m} 2 \mathrm{~g}$ preserves the unique properties of a given MRI scan, additional care must be taken to determine whether the connectomes generated by $\mathrm{m} 2 \mathrm{~g}$ even conform with basic phenomena that are observed in the brain. One 
such process to do this involves observation of either to the physical or functional connection between different regions of the brain. Three simple categories for these regions are ipsilateral (connections that stay within the left or right hemispheres), contralateral (across hemispheres), or homotopic (from one region of the brain to the same region on the other hemisphere). Using the edge weights from the structural connectomes generated by $\mathrm{m} 2 \mathrm{~g}$, the percent of edges that fell into each of the three connection types was calculated, with the mean and standard deviation were found for each dataset. This process was performed for three parcellations which had clearly-defined homotopic regions of interest, namely the DKT, Hammersmith, and AAL atlases. For the functional connectomes generated, the Pearson's correlation between regions of interest was used as a metric. The mean and standard deviation of each of the three connection types was found for each dataset (Figure 4). This process was also performed for the same three parcellations, which had similarly consistent results.

5 Ethical Compliance We complied with all relevant ethical regulations. This study reused publicly available data acquired at many different institutions. Protocols for all of the original studies were approved by the corresponding ethical boards.

6 Software Availability All of our code is available from our website, http://m2g.io, and has been deposited into our public github repository, https://github.com/neurodata/m2g, and published with a DOI, https://doi.org/10.5281/zenodo.1161284, under the Apache License 2.0.

7 Data Availability The data derivatives that support the findings of this study are available from our website, http://m2g.io, under a (ODC-By) v1.0 license.

Acknowledgements The authors from JHU are grateful for the support by the XDATA program of the Defense Advanced Research Projects Agency (DARPA) administered through Air Force Research Laboratory contract FA8750-12-2-0303; DARPA SIMPLEX program through SPAWAR contract N66001-15-C-4041; DARPA GRAPHS contract N66001-14-1-4028; National Science Foundation grant 1649880, and the Kavli Foundation for their support. Dr. Xi-Nian Zuo received funding support in China from the National Basic Research (973) Program (2015CB351702), the National R\&D Infrastructure and Facility Development Program "Fundamental Science Data Sharing Platform" (DKA2017-12-02-21), the Natural Science Foundation of China (81471740, 81220108014) and Beijing Municipal Science and Tech Commission (Z161100002616023, Z161100000216152). Dr. Calhoun received funding from the NIH (P20GM103472 and R01EB020407) and the NSF (grant 1539067).

Author Information $\mathrm{RL}^{1,3, *}, \mathrm{AL}^{1,3}, \mathrm{GK}^{1,2}$, $\mathrm{EWB}^{3,16}, \mathrm{WG}^{4}, \mathrm{VCh}^{1,3}, \mathrm{DM}^{5}, \mathrm{SR}^{6}, \mathrm{XZ}^{7,8,9,10}, \mathrm{DSM}^{11}, \mathrm{RCC}^{12,13}$, $\mathrm{CEP}^{3,14}, \mathrm{BC}^{16}, \mathrm{RJ}^{6}, \mathrm{VCa}^{15}, \mathrm{BC}^{16}, \mathrm{RB}^{5}, \mathrm{MPM}^{9}, \mathrm{JTV}^{1,3,5,9,17, \dagger}$ :

* corresponding author.

${ }^{1}$ Department of Biomedical Engineering, Johns Hopkins University, Baltimore, MD, USA.

2 Department of Biomedical Engineering, McGill University, Baltimore, MD, USA.

${ }^{3}$ Center for Imaging Science, Johns Hopkins University, Baltimore, MD, USA.

${ }^{4}$ Johns Hopkins University Applied Physics Laboratory, Laurel, MD, USA.

${ }^{5}$ Department of Computer Science, Johns Hopkins University, Baltimore, MD, USA.

${ }^{6}$ Department of Psychology, University of New Mexico, Albuquerque, NM, USA.

7 Department of Psychology, University of Chinese Academy of Sciences (CAS), Beijing, China.

${ }^{8}$ CAS Key Laboratory of Behavioral Science, Beijing, China.

${ }^{9}$ Research Center for Lifespan Development of Mind and Brain (CLIMB), CAS Institute of Psychology, Beijing, China.

${ }^{10}$ Magnetic Resonance Imaging Research Center (MRIRC), CAS Institute of Psychology, Beijing, China.

${ }^{11}$ Department of Human Cognitive and Brain Sciences, Max-Planck Institute, Munich, Germany.

${ }^{12}$ Child Mind Institute, New York, NY, USA.

${ }^{13}$ Dell Medical School, University of Texas at Austin, Austin, TX, USA.

${ }^{14}$ Department of Statistics, Johns Hopkins University, MD, USA. 
bioRxiv preprint doi: https://doi.org/10.1101/2021.11.01.466686; this version posted November 3, 2021. The copyright holder for this preprint (which was not certified by peer review) is the author/funder, who has granted bioRxiv a license to display the preprint in perpetuity. It is made available under aCC-BY-NC-ND 4.0 International license.

${ }^{15}$ Department of Biomedical Engineering, University of New Mexico, Albuquerque, NM, USA.

${ }^{16}$ Department of Biostatistics, Johns Hopkins University, MD, USA.

17 Institute for Computational Medicine, Johns Hopkins University, Baltimore, MD, USA.

$\dagger$ is the corresponding author: 〈jovo@jhu.edu〉.

CoRR Members Jeffrey S. Anderson, Pierre Bellec, Rasmus M. Birn, Bharat B. Biswal, Janusch Blautzik, John C.S. Breitner, Randy L. Buckner, F. Xavier Castellanos, Antao Chen, Bing Chen, Jiangtao Chen, Xu Chen, Stanley J. Colcombe, William Courtney, Adriana Di Martino, Hao-Ming Dong, Xiaolan Fu, Qiyong Gong, Krzysztof J. Gor-I golewski, Ying Han, Ye He, Yong He, Erica Ho, Avram Holmes, Xiao-Hui Hou, Jeremy Huckins, Tianzi Jiang, Yi Jiang, William Kelley, Clare Kelly, Margaret King, Stephen M. LaConte, Janet E. Lainhart, Xu Lei, HuiJie Li, Kaiming Li, Kuncheng Li, Qixiang Lin, Dongqiang Liu, Jia Liu, Xun Liu, Guangming Lu, Jie Lu, Beatriz Luna, Jing Luo, Daniel Lurie, Ying Mao, Andrew R. Mayer, Thomas Meindl, Mary E. Meyerand, Weizhi Nan, Jared A. Nielsen, David OConnor, David Paulsen, Vivek Prabhakaran, Zhigang Qi, Jiang Qiu, Chunhong Shao, Zarrar Shehzad, Weijun Tang, Arno Villringer, Huiling Wang, Kai Wang, Dongtao Wei, Gao-Xia Wei, Xu-Chu Weng, Xuehai Wu, Ting Xu, Ning Yang, Zhi Yang, Yu-Feng Zang, Lei Zhang, Qinglin Zhang, Zhe Zhang, Zhiqiang Zhang, Ke Zhao, Zonglei Zhen, Yuan Zhou, Xing-Ting Zhu.

\section{References}

[1] E J.R. van Beek et al. Value of mri in medicine: More than just another test? J Magn Reson Imaging, 49:e14-e25, 2018. doi: $10.1002 /$ jmri.26211.

[2] Eleftherios Garyfallidis, Matthew Brett, Bagrat Amirbekian, Ariel Rokem, Stefan Van Der Walt, Maxime Descoteaux, and lan Nimmo-Smith. Dipy, a library for the analysis of diffusion mri data. Frontiers in neuroinformatics, 8:8, 2014.

[3] Shan Jiang, Pengfei Zhang, Tong Han, Weihua Liu, and Meixia Liu. Tri-linear interpolation-based cerebral white matter fiber imaging. Neural Regen Res, 8:2155-2164, 2015. doi: 10.3969/j.issn.1673-5374.2013.23.005.

[4] Amir Pasha Mahmoudzadeh and Nasser H Kashou. Evaluation of interpolation effects on upsampling and accuracy of cost functions-based optimized automatic image registration. International Journal of Biomedical Imaging, 2013. doi: $10.1155 / 2013 / 395915$.

[5] Shangsi Wang, Zhi Yang, Michael Milham, Cameron Craddock, Xi-Nian Zuo, Carey E. Priebe, and Joshua T. Vogelstein. Optimal experimental design for generating reference connectome datasets. In Organization for Human Brain Mapping, 21st Annual Meeting, 2015.

[6] Bennett A Landman, Alan J Huang, Aliya Gifford, Deepti S Vikram, Issel Anne L Lim, Jonathan AD Farrell, John A Bogovic, Jun Hua, Min Chen, Samson Jarso, et al. Multi-parametric neuroimaging reproducibility: a 3-t resource study. Neuroimage, 54(4):2854-2866, 2011.

[7] Xi-Nian Zuo, Jeffrey S Anderson, Pierre Bellec, Rasmus M Birn, Bharat B Biswal, Janusch Blautzik, John CS Breitner, Randy L Buckner, Vince D Calhoun, F Xavier Castellanos, et al. An open science resource for establishing reliability and reproducibility in functional connectomics. Scientific data, 1:140049, 2014.

[8] A. Di Martino, CG Yan, Q Li, E. Denio, FX Castellanos, K Alaerts, JS Anderson, M. Assaf, M Bookheimer, and et. al. The autism brain imaging data exchange: towards a large-scale evaluation of the intrinsic brain architecture in autism. Mol Psychiatry, 19:659-667, 2014. doi: 10.1038/mp.2013.78.

[9] A. Di Martino, David O'Conner, Bosi Chen, Kaat Alaerts, Jeffrey S. Anderson, and et. al. Enhancing studies of the connectome in autism using the autism brain imaging data exchange ii. Sci Data, 4, 2017. doi: 10.1038/sdata.2017.10.

[10] R.M. Lawrence, E.W Bridgeford, P.E. Myers, and et al. Standardizing human brain parcellations. Sci Data, 8, 2021. doi: $10.1038 / \mathrm{s} 41597-00849-3$.

[11] Richard F Betzel, Alessandra Griffa, Patric Hagmann, and Bratislav Misic. Distance-dependent consensus thresholds for generating group-representative structural brain networks. 3:475-496, 2019. doi: 10.1162/netn_a_00075.

[12] Bratislav Misic and et al. Cooperative and competitive spreading dynamics on the human connectome. Neuron, 86: 1518-1529, 2015. doi: 10.1016/j.neuron.2015.05.035.

[13] R.S. et al. Desikan. An automated labeling system for subdividing the human cerebral cortex on mri scans into gyral based regions of interest. Neuroimage, 31:968-980, 2006. doi: 10.1016/j.neuroimage.2006.01.021.

[14] John D Lewis, Rebecca J Theilmann, Martin I Sereno, and Jeanne Townsend. The relation between connection length and degree of connectivity in young adults: a dti analysis. Cereb Cortex, 19(3):554-563, 2009. doi: 10.1093/crecor/ bhn105.

[15] David E Stark, Daniel S Margulies, Zarrar E Shehzad, Philip Reiss, A. M. Clare Kelly, Lucina Q Uddin, Dylan G Gee, Amy K Roy, Marie T Banich, F Xavier Castellanos, and Michael P Milham. Correction for david et al., regional variation in interhemispheric coordination of intrisic hemodynamic fluctuations. 29, 2009. ISSN 0270-6474. doi: 10.1423/ JNEUROSCI.4544-08.2008.

[16] Bharat B Biswal, Clare Kelly, David E Stark, Daniel S Margulies, Zarrar Shehzad, Lucina Q Uddin, Donald F Klein, Marie T Banich, F Xavier Castellanos, and Michael P Milham. Low frequency fluctuations reveal integrated and segre- 
bioRxiv preprint doi: https://doi.org/10.1101/2021.11.01.466686; this version posted November 3, 2021. The copyright holder for this preprint (which was not certified by peer review) is the author/funder, who has granted bioRxiv a license to display the preprint in perpetuity. It is made available under aCC-BY-NC-ND 4.0 International license.

gated processing among the cerebral hemispheres. pages 517-527, 2011. doi: 10.1016/j.neuroimage.2010.05.073.

[17] Xi-Nian Zuo, Clare Kelly, Adriana Di Martino, Maarten Mennes, Daniel S Marguile, Saroja Bangaru, Rebecca Grzadzinski, Alan C Evans, Yu-Feng Zang, F Xavier Castellanos, and Michael P Milham. Growing together and growing apart: Regional and sex differences in the lifespan developmental trajectories of functional homotopy. 30:15034-15043, 2010. doi: 10.1523/JNEUROSCI.2612-10.2010.

[18] N. Tzourio-Mazoyer and et al. Automated anatomical labeling of activations in spm using a macroscopic anatomical parcellation of the mri single-subject brain. Neuroimage, 15:273-289, 2002. doi: 10.1006/nimg.2001.0978.

[19] S. loannis and et.al. Automatic segmentation of a brain mris of 2-year-olds into 83 regions of interest. Neurolmage, 40: 672-684, 2008.

[20] Wilhelm Kirch, editor. Pearson's Correlation Coefficient, pages 1090-1091. Springer Netherlands, Dordrecht, 2008. ISBN 978-1-4020-5614-7. doi: 10.1007/978-1-4020-5614-7_2569. URL https://doi.org/10.1007/978-1-4020-5614-7_ 2569.

[21] Mirza Faisal Beg, Michael I Miller, Alain Trou, and Laurent Younes. Computing Large Deformation Metric Mappings via Geodesic Flows of Diffeomorphisms. International Journal of Computer Vision, 61(2):139-157, 2005. doi: 10.1023/B:VISI.0000043755.93987.aa. URL https://www.researchgate.net/profile/Alain__\}Trouve/publication/

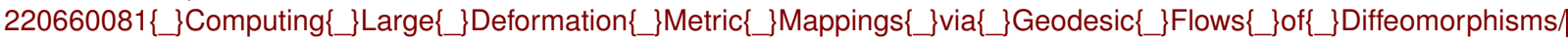
links/0912f5076f959f39ee000000.pdf.

[22] T.E.J. Behrens, M.W. Woolrich, Jenkinson, M. H. Johansen-Berg, R.G. Nunes, S. Clare, P.M. Matthews, J.M. Brady, and S.M. Smith. Characterization and Propegation of Uncertainty in Diffusion Weighted MR images. Technical report, 2003. URL http://www.fmrib.ox.ac.uk/datasets/techrep/tr03tb1/tr03tb1/.

[23] Jean-Philippe Fortin, Drew Parker, Birkan Tunç, Takanori Watanabe, Mark A Elliott, Kosha Ruparel, David R Roalf, Theodore D Satterthwaite, Ruben C Gur, Raquel E Gur, Robert T Schultz, Ragini Verma, and Russell T Shinohara. Harmonization of multi-site diffusion tensor imaging data. Neuroimage, 161:149-170, August 2017.

[24] Jeffrey T. Leek and John D. Storey. Capturing Heterogeneity in Gene Expression Studies by Surrogate Variable Analysis. PLoS Genetics, 3(9):e161, 2007. ISSN 1553-7390. doi: 10.1371/journal.pgen.0030161. URL http://dx.plos.org/10. 1371/journal.pgen.0030161.

[25] K.J. Gorgolewski et al. The brain imaging data structure, a format for organizing and describing outputs of neuroimaging experiments. Sci Data, 3:160044, 2016. doi: 10.1038/sdata.2016.44.

[26] Stephen M Smith et al. Advances in functional and structural MR image analysis and implementation as FSL. Neurolmage, 23 Suppl 1:S208-19, jan 2004. ISSN 1053-8119. URL http://www.ncbi.nlm.nih.gov/pubmed/15501092.

[27] Mark W Woolrich et al. Bayesian analysis of neuroimaging data in FSL. Neurolmage, 45(1 Suppl):S173-86, mar 2009. ISSN 1095-9572. URL http://www.sciencedirect.com/science/article/pii/S1053811908012044.

[28] Mark Jenkinson et al. FSL. Neurolmage, 62(2):782-90, aug 2012. ISSN 1095-9572. URL http://www.ncbi.nlm.nih.gov/ pubmed/21979382.

[29] G. Grabner, A.L. Janke, M.M. Budge, D. Smith, J. Pruessner, and D.L. Collins. Symmetric atlasing and model based segmentation: an application to the hippocamus in older adults. Med Image Comput Comput Assist Interv Int Conf Med Image Comput Comput Assist Interv, 9:58-66, 2006. doi: 10.1007/11866763_8.

[30] Matthew Brett, Christopher J. Markiewicz, Michael Hanke, Marc-Alexandre Côté, Ben Cipollini, Paul McCarthy, Dorota Jarecka, Christopher P. Cheng, Yaroslav O. Halchenko, Michiel Cottaar, Eric Larson, Satrajit Ghosh, Demian Wassermann, Stephan Gerhard, Gregory R. Lee, Hao-Ting Wang, Erik Kastman, Jakub Kaczmarzyk, Roberto Guidotti, Or Duek, Jonathan Daniel, Ariel Rokem, Cindee Madison, Brendan Moloney, Félix C. Morency, Mathias Goncalves, Ross Markello, Cameron Riddell, Christopher Burns, Jarrod Millman, Alexandre Gramfort, Jaakko Leppäkangas, Anibal Sólon, Jasper J.F. van den Bosch, Robert D. Vincent, Henry Braun, Krish Subramaniam, Krzysztof J. Gorgolewski, Pradeep Reddy Raamana, Julian Klug, B. Nolan Nichols, Eric M. Baker, Soichi Hayashi, Basile Pinsard, Christian Haselgrove, Mark Hymers, Oscar Esteban, Serge Koudoro, Fernando Pérez-García, Nikolaas N. Oosterhof, Bago Amirbekian, Ian Nimmo-Smith, Ly Nguyen, Samir Reddigari, Samuel St-Jean, Egor Panfilov, Eleftherios Garyfallidis, Gael Varoquaux, Jon Haitz Legarreta, Kevin S. Hahn, Oliver P. Hinds, Bennet Fauber, Jean-Baptiste Poline, Jon Stutters, Kesshi Jordan, Matthew Cieslak, Miguel Estevan Moreno, Valentin Haenel, Yannick Schwartz, Zvi Baratz, Benjamin C Darwin, Bertrand Thirion, Carl Gauthier, Dimitri Papadopoulos Orfanos, Igor Solovey, Ivan Gonzalez, Jath Palasubramaniam, Justin Lecher, Katrin Leinweber, Konstantinos Raktivan, Markéta Calábková, Peter Fischer, Philippe Gervais, Syam Gadde, Thomas Ballinger, Thomas Roos, Venkateswara Reddy Reddam, and freec84. nipy/nibabel: 3.2.1, November 2020. URL https://doi.org/10.5281/zenodo.4295521.

[31] AFNI: Software for Analysis and Visualization of Functional Magnetic Resonance Neuroimages. Computers and Biomedical Research, 29(3):162-173, jun 1996. ISSN 0010-4809. doi: 10.1006/CBMR.1996.0014. URL http: //www.sciencedirect.com/science/article/pii/S0010480996900142.

[32] R Cameron Craddock, G Andrew James, Paul E Holtzheimer, Xiaoping P Hu, and Helen S Mayberg. A whole brain fmri atlas generated via spatially constrained spectral clustering. Human brain mapping, 33(8):1914-1928, 2012.

[33] Yashar Behzadi, Khaled Restom, Joy Liau, and Thomas T. Liu. A component based noise correction method (compcor) for bold and perfusion based fmri. Neurolmage, 37(1):90-101, 2007. ISSN 1053-8119. doi: https://doi.org/10.1016/ j.neuroimage.2007.04.042. URL https://www.sciencedirect.com/science/article/pii/S1053811907003837. 